Teknokultura. Revista de Cultura Digital y Movimientos Sociales

ISSNe: $1549-2230$

http://dx.doi.org/10.5209/TEKN.65189

\title{
Precariedad e inestabilidad: contradicciones en el trabajo en las plataformas de reparto de comida
}

\author{
Francisco Fernández-Trujillo Moares ${ }^{1}$
}

Recibido: 22 de julio 2019 / Aceptado: 14 de diciembre 2019 Open peer reviews

Resumen. Las plataformas digitales han aparecido y se han desarrollado en un marco de crisis económica que ha supuesto la profundización de diversas formas de desigualdad, precarización, desmejora de las condiciones de vida para amplios grupos de población y ha generado cambios de gran envergadura en las economías. Esto habría supuesto que su expansión responda a una serie de características en concordancia con dicha situación. Así, enmarcado en un momento de gran expansión de las economías digitales y el capitalismo de plataforma, en este texto se pretende hacer una aproximación a algunas de las claves explicativas de los elementos de un tipo concreto de plataformas digitales: las plataformas de reparto de comida. Aquí se analizan algunas de las cuestiones que han supuesto, desde su aparición, mayor controversia y discusión: momento de emergencia, innovación, flexibilidad en el trabajo o su papel en el mercado laboral. Para ello se analizan algunos de los discursos presentados por las propias plataformas digitales de reparto y los discursos de los repartidores y repartidoras de estas plataformas

Palabras clave: crisis; economías digitales; plataformas digitales; precariedad; trabajo.

\section{[en] Precarity and instability: contradictions in the work on food delivery platforms}

\begin{abstract}
Digital platforms have appeared and developed in a framework of economic crisis that has led to the deepening of various forms of inequality, precariousness, deterioration of living conditions for large sections of the population. These platforms have also generated far-reaching changes in economies. This would have meant that its expansion responds to a series of characteristics in accordance with that situation. Attending to the great expansion of digital economies and platform capitalism, this text intends to make an approximation to some of the explanatory keys of the elements of a specific type of digital platforms: food delivery platforms. Here some of the issues that have arisen since their greatest controversy and discussion is analyzed: the moment of emergency, innovation, flexibility at work or their role in the labour market. To this end, some of the discourses presented by the digital delivery platforms themselves and the discourses of the riders of these platforms are analyzed.
\end{abstract}

Keywords: crisis; digital economies; digital platforms; precarity; labour.

Sumario. 1. Introducción. 2. Concepción y contextualización de la emergencia de la economía de plataforma. 3. Objetivo y planteamiento de la investigación. 4. Resultados. Los discursos en el trabajo en las plataformas de reparto. 5. Conclusiones. 6. Referencias.

Cómo citar: Fernández-Trujillo Moares, F. (2020). Precariedad e inestabilidad: contradicciones en el trabajo en las plataformas de reparto de comida. Teknokultura. Revista de Cultura Digital y Movimientos Sociales, 17(1), 35-45.

\section{Introducción}

En la última década se ha dado la proliferación, expansión por diferentes sectores y el desarrollo de diversas plataformas que han supuesto una gran incidencia en las formas de consumo, trabajo y prestación de servicios en la economía. El desarrollo de estas plataformas ha sido desigual en los diferentes sectores, y aunque es todavía relativamente reducido el número de personas que desarrollan una actividad laboral, especialmente las que lo hacen en exclusiva, y la cantidad de transacciones que se dan en estas plataformas están todavía alejadas de las de las formas más tradicionales de economía, sin embargo, existiría cierto consenso en su rápida expansión (Howcroft y Bergvall-Kåreborn, 2019; Vallas, 2019).

Cada vez un número mayor de tareas y actividades son realizadas a través de plataformas digitales, prestando servicios populares y conocidos en España como el transporte de personas (Uber o Cabify) o el reparto y la mensajería de comida u otros productos (Deliveroo, Glovo o UberEats), pero también otros cada vez más utilizados como el cuidado de niños y niñas, paseo de perros, tours para turistas o servicios legales (Florisson y Mandl, 2018, p. 1).

\footnotetext{
Universidad Nacional de Educación a Distancia (UNED) (España)

E-mail: franciscoftm@poli.uned.es
} 
Como han señalado diferentes investigaciones en los últimos años, la aparición de este tipo de plataformas implica múltiples riesgos y amenazas posibles, como la carencia de transparencia de éstas en la utilización de datos (Vandeale, 2018:5), los problemas de adaptación y ocasionales ausencias de marcos regulatorios en distintos aspectos de las plataformas (Berins, Dubal y Carter, 2017; Florisson y Mandl, 2018; Vandaele, 2018), la situación impositiva y tributaria de las plataformas (Miguélez, 2018) o la limitación de derechos de quienes trabajan en éstas (De Stefano, 2016; Vandaele, 2018; Zamponi, 2018).

En este texto se abordan algunos de los aspectos considerados clave en la emergencia y desarrollo de estas empresas partiendo de que la aparición de estas plataformas se da en un momento específico, como es la crisis económica, que habría supuesto que se desarrollen con ciertas características concretas, prestando este estudio especial atención a la contraposición entre flexibilidad frente a seguridad y a la precariedad asociada a este tipo de plataformas.

Por ello este texto está compuesto de una primera parte en la que se hace una aproximación al tipo concreto de plataformas que son estudiadas, las plataformas de reparto de comida, y se sitúan los que son considerados como principales elementos contextuales que explican las características de su desarrollo y crecimiento en España. En una segunda parte, se atiende a algunas cuestiones clave en la consolidación de estas plataformas, como son la crisis económica iniciada en 2008 como momento de aparición y crecimiento de las plataformas de reparto; la discusión en torno a lo innovador de estas plataformas; el papel que ha supuesto en los últimos años en el mercado de trabajo y las implicaciones que tendría sobre las condiciones laborales; y la flexibilidad como cuestión en discusión por quienes están involucrados e involucradas en el conflicto. Para ello se analizan los discursos extraídos de doce entrevistas realizadas a repartidores y repartidoras en Madrid.

Así, en esta investigación se pretende responder y explicar las condiciones y características de las formas de trabajo en las grandes plataformas de reparto encontradas en los discursos de repartidores y repartidoras de la llamada gig economy. Esta cuestión es abordada desde dos elementos vertebrados por la contraposición entre la flexibilidad y la seguridad en el trabajo: la generación de un escenario en el que el mercado laboral estaría fuertemente marcado por la precariedad $\mathrm{y}$, en relación a esto, por la profundización de esta realidad de precariedad a través de formas de gestión y organización en el trabajo que son presentados como innovación, transformación y adaptación a las nuevas realidades desde quienes lo implantan, las grandes plataformas de reparto de comida.

\section{Concepción y contextualización de la emergencia de la economía de plataforma}

\subsection{Una breve aproximación a la economía de plataforma}

Es habitual encontrar no solo en el ámbito periodístico, sino también en el ámbito académico, diferentes maneras de referirse al tipo de economías derivadas de la aparición de las plataformas digitales, a las mismas plataformas y a sus categorías. En ocasiones esto puede llevar a que se produzcan algunas confusiones terminológicas y conceptuales, al no estar del todo claras a qué tipo de actividades, relaciones y qué procesos y actores están implicados en el desarrollo de los argumentos planteados. Es por esto que en esta investigación se presenta una aproximación a la terminología utilizada.

Para ello es útil la investigación para Eurofound de Florisson y Mandl (2018, p. 2, nuestra traducción) en la que definen el trabajo de plataforma de una forma concisa y esquemática, planteando que éste es:

[U]na forma de empleo que utiliza una plataforma para permitir a las organizaciones o individuos acceder a otras organizaciones o individuos para resolver problemas específicos o para proporcionar servicios específicos a cambio de un pago. En consecuencia, la característica principal del trabajo de plataforma es que enlace la oferta y la demanda de mano de obra remunerada. Las principales características de la plataforma trabajo [...] son: Trabajo remunerado organizado a través de plataformas: Tres partes implicadas: plataforma, cliente, trabajador; El objetivo es llevar a cabo tareas específicas o resolver problemas específicos; Forma de subcontratación/contratación; Desglose de 'puestos de trabajo' en 'tareas'; Servicios bajo demanda.

Es necesario tener en cuenta que las plataformas digitales se han implantado en los últimos años de manera cada vez más contundente, introduciéndose en un número cada vez mayor de sectores e implicando a un creciente número de servicios y actividades (Florisson y Mandl, 2018; Howcroft. y BergvallKåreborn, 2019; Vallas, 2019). Aunque los casos más conocidos y presentes en el debate sobre las plataformas digitales son el transporte de pasajeros o reparto de comida a domicilio. En los últimos años en España los procesos de transformación y problemas asociados al trabajo de plataforma más conocidas han sido el conflicto de competencia entre las plataformas de VTC y el sector del taxi y la precariedad asociada a las plataformas de reparto de comida a domicilio. No obstante la realidad es que la implantación de las plataformas se hace patente de manera creciente en un número cada vez mayor de sectores. Ejemplo de ello es la creciente popularidad de las plataformas de diferentes formas de cuidado como Cuideo o Yoopies, de actividades profesionales de diseño, programación o análisis de datos como Upwork and 99 Designs, de trabajos domésticos como Hilfr, Helpling o Listminut, entre otras muchas. 
Buena parte de las dificultades a la hora de delimitar y definir el trabajo en las plataformas digitales se debe en numerosas ocasiones a que éstas están presentes no solo en un cada vez mayor número de sectores, actividades y servicios diferentes, sino también a que tienen asociadas múltiples formas de trabajo con un carácter atípico, como son diferentes formas de contrataciones de servicios, trabajo informal, trabajo compartimentado, teletrabajo, crowdwork, trabajo llevado a cabo por autónomos o autónomas (Garben, 2017, p. 3).

A la hora de mirar las plataformas digitales debemos tener en cuenta las distintas formas de plataformas y atender a algunas de las categorizaciones que se han realizado en los estudios recientes. Si bien encontramos algunas tipologías como las de Howcroft y Bergvall-Kåreborn (2019) basadas en el tipo de remuneración y quién inicia el contacto para la realización de tarea o algunas clasificaciones de Srnicek (2017) en su investigación sobre las plataformas digitales atendiendo a diferentes características, como actores que participan en el proceso de resolución de tareas o el rol de la plataforma en el proceso, para este análisis es útil para enmarcar las plataformas de reparto de comida la clasificación de Vallas (2019). Ésta estaría basada en una distinción entre una primera dimensión en función de la dispersión espacial diferenciando entre crowdworking y gig economy y otra dimensión en relación al nivel de habilidades necesarias y complejidad de las tareas a realizar requeridas por las plataformas. Sin embargo, también es necesario tener en cuenta que dichas habilidades requeridas son también objeto de crítica por algunos y algunas profesionales del ámbito del reparto y la mensajería, denunciando una desprofesionalización del sector a partir de la llegada de las plataformas digitales.

$\mathrm{Y}$ es que, como plantea De Stefano, en el trabajo bajo demanda a través de apps (work on-demand via apps) las actividades que son llevadas a cabo están relacionadas con formas tradicionales de empleo o actividades que se venían desarrollando de manera previa a la emergencia de las plataformas digitales, como el transporte, la limpieza, realización de recados de distinto tipo, pero también actividades de oficina. Las plataformas basan su negocio en la organización de estas tareas a través de la aplicación, estableciendo criterios de calidad mínimos y gestionando la selección del personal y la fuerza de trabajo (De Stefano, 2016, p. 5). Esto es una cuestión fundamental a la hora de aproximarse al trabajo de plataforma, ya que permite entender las plataformas de trabajo bajo demanda más como herramienta de organización del trabajo en distintas formas de actividad laboral que como la aparición de una serie de nuevos empleos (De Stefano, 2016:1).

En relación al objeto que ocupa esta investigación, como señala la literatura (De Stefano, 2016; Miguélez, 2018; Srnicek, 2017), el trabajo de reparto en las plataformas son actividades laborales tradicionales organizadas a través de plataformas digitales.
Esto también se ve reflejado en que la ejecución es bastante similar con respecto a lo que señalan los repartidores y las repartidoras que habían trabajado en esta actividad con anterioridad y los que trabajan para otro tipo de empresas o entidades que no están mediadas por las plataformas, aunque con condiciones distintas.

En este sentido Gil (2018) señalaría que la utilización del apelativo colaborativo sería más un reclamo que una realidad de este tipo de plataformas. En el mismo sentido, una de las imágenes más ilustrativas propuesta por quienes ponen en cuestión el carácter colaborativo de las compañías de reparto sería la de la caja negra o "black box" (Conaty, Bird y Ross, 2018). En ésta la plataforma actuaría como una caja negra a través de la que tendrían que pasar todas las comunicaciones entre quienes están involucrados en el proceso (restaurantes, repartidores y repartidoras y consumidores y consumidoras finales) bloqueando cualquier comunicación directa, siendo esta su vía de extracción de valor (Conaty, Bird y Ross, 2018, p. 7). Así, para de De Rivera, Gordo y Cassidy (2017, pp. 23-26) algunas de los modelos generados a partir del desarrollo y concepción de la economía colaborativa se han constituido como un reclamo y estrategia comercial insertas en las lógicas neoliberales.

Sin embargo, otros autores han planteado la economía colaborativa desde una perspectiva crítica y alternativa a la ofrecida por las grandes plataformas y compañías cubiertas bajo el paraguas de lo colaborativo. Para Lietaert (2017) se habrían podido crear espacios en los mercados alternativos gracias a la aparición de internet y el uso como herramienta para movimientos sociales, dando un uso determinado a los desarrollos tecnológicos para la creación de alternativas económicas más horizontales y solidarias. En esta línea, en el informe para Trades Union Congress (TUC), es planteada la constitución de cooperativas como solución a la precariedad en el marco de la expansión y el desarrollo de las plataformas digitales (Conaty, Bird y Ross, 2018). En este informe, se señalan ejemplos de experiencias cooperativas en este ámbito, en el que el uso tecnológico de apps tiene gran importancia, como las cooperativas de taxi (Conaty, Bird y Ross, 2018, pp. 7-9, 12-13). Bajo esta misma premisa es desarrollado el concepto de cooperativismo de plataforma, dada la utilización de tecnologías similares a la de las grandes plataformas ofreciendo servicios semejantes, pero bajo lógicas de relaciones sociales y productivas diferentes (Gil, 2018, p. 56; Scholz, 2016, pp. 2, 14).

\subsection{Flexibilidad y seguridad: el mercado laboral tras la crisis}

Durante la crisis en España se habrían dado diferentes procesos de transformación de la economía que habrían afectado y estarían relacionados con la proliferación y desarrollo de la economía de plataformas. En este sentido, la tendencia que caracterizaría la situación del em- 
pleo que permite comprender la coyuntura actual tendría un carácter y sería una tendencia de la economía global. Como plantea Srnicek (2017, pp. 35-36):

Con el colapso del comunismo ha habido una tendencia de largo plazo tanto hacia una mayor proletarización como hacia cantidades mayores de población excedente. Gran parte del mundo recibe hoy en día un ingreso reculado por el mercado a través de trabajo precario e informal. Este ejercicio de reserva se expandió significativamente después de la crisis de 2008.

Asimismo, durante la crisis global se habría generado un marco de vulnerabilidad para los distintos sistemas de bienestar y protección para buena parte de los marcos legislativos y jurídicos de defensa de las condiciones vitales para buena parte de la población. La aplicación de las políticas de austeridad y el deterioro de las economías nacionales como consecuencia de la crisis habrían desmantelado en parte la capacidad de los Estados para controlar las transformaciones que se habrían estado dando en la economía (Laval y Dardot, 2013, pp. 17-23). Es decir, se habría generado un clima de desprotección ante las transformaciones que se habrían venido dando durante el periodo iniciado después de 2008.

$\mathrm{Y}$ es que es necesario tener en cuenta que durante la crisis económica se ha generado un escenario de vulnerabilidad social en el que se ha producido un retroceso en los niveles de bienestar y de las condiciones de vida para parte de la población, en el que el acceso a algunos aspectos sociales clave como el trabajo se han visto fuertemente afectados. Esto habría abierto la posibilidad a que se dieran las condiciones en la década presente donde la digitalización de la economía y la llamada "economía del dato" habrían jugado un papel fundamental (Sadin, 2018, pp. 26-27).

Como señala Nick Srnicek en una de las líneas de desarrollo más importantes de su libro Capitalismo de plataformas, en el que plantea que "las distintas crisis establecieron los precedentes para la actual economía post-2008 y en él intenta historizar las tecnologías emergentes como resultado de tendencias económicas más profundas" (Srnicek, 2018, p. 14).

Uno de los problemas concretos sería la tendencia entre las empresas a las externalizaciones y la provisión de servicios de terceros, que habitualmente serían provistos por personal dado de alta como autónomo y pequeñas empresas (Calderón y López Calle, 2010). Actividades habitualmente incorporadas a las plantillas de las empresas habrían pasado a ser llevadas a cabo por otras vías.

Entre las plataformas digitales, bajo la categoría que denomina Srnicek "plataformas austeras", aquellas en las que se pueden encontrar usuarios, clientes y trabajadores, pero en las que su activo más importante sería la plataforma de software y análisis de datos, operando a través de un modelo "hiperterciarizado" (Srnicek, 2017, pp. 71-72).

Otro habría sido el especial deterioro en Europa en general (Bouffartigue, 2015) y en España en particular (Santos y Martín, 2012) de las condiciones labora- les para los grupos más vulnerables de los mercados laborales. Siendo aquí de especial importancia la población migrante y los jóvenes, alcanzando tasas de desempleo superiores al 50\% entre los menores de 30 años. La contratación y las formas de empleo generadas habrían estado caracterizadas por bajos salarios y contrataciones a tiempo parcial (Santos y Martín, 2012). Se habrían ido generando así dificultades para la entrada en el mercado laboral para amplias capas de población, con cada vez una menor exigencia para los puestos de trabajo ofertados debido a la necesidad de incorporarse a aquel.

Otro de los problemas derivados de esta situación es un panorama en el que el sindicalismo no podría realizar sus funciones de representación y defensa de las condiciones de trabajo con los recursos tradicionales o habituales debido a las nuevas formas de trabajo atípicas generadas durante la crisis como han señalado Calderón y López Calle (2010). Éstos hacen referencia a diversas formas de trabajo diferentes a los asalariados y las asalariadas tradicionales, quienes se ven afectados indirectamente por los resultados de la negociación colectiva. Entre estas categorías se encontrarías becarios y becarias, autónomos y autónomas o quienes realizan trabajado voluntario (Calderón y López Calle, 2010, p. 6).

Con la expansión de las plataformas durante la crisis global de 2008 numerosos aspectos del desarrollo político, social y económico se han visto profundamente afectados y alterados. En este momento se implantaba la idea de una necesidad de cambio y transformación en los procesos económicos que habrían supuesto la emergencia de nuevas formas de capitalismo o de reorganización de las formas de la economía, como la economía de plataforma. Esto se daría en el marco de lo que autores como Srnicek (2018, p. 39) consideraría una estrategia de reestructuración del capitalismo como respuesta a la crisis.

Como ha sido señalado, las plataformas digitales se han instalado y se han desarrollado en muy diversos sectores, sin embargo, es necesario tener en cuenta que, como señala Miguélez (2018, p. 151), el trabajo de plataforma responde a la realidad y a las características del mercado laboral existente, especialmente aquellos marcados por altos niveles de precariedad, así:

[G]ran parte del empleo creado se está dando básicamente en los sectores y las ocupaciones que corresponden a la estructura productiva dominante en las últimas décadas: hostelería, restauración, comercio, servicios sociales, construcción son los sectores que destacan [...] pero siempre con características de cualificación y profesionalización poco exigentes.

En este contexto, el ámbito del trabajo ha sido uno de los más afectados por la crisis, dándose grandes tasas de desempleo y un aumento de la precariedad laboral de manera generalizada, aunque mostrando especial contundencia entre los sectores más vulnerables del mercado laboral.

Asimismo, Standing (2011) expondría que se habría generado una nueva realidad para grandes secto- 
res de la población caracterizados por la precariedad. El momento de desarrollo y proliferación de las plataformas digitales de trabajo y la gig economy sería en buena medida la culminación del proceso generado en los 70 en el que se ponían en marcha los discursos y lógicas neoliberales de la flexibilidad en el mercado laboral (Standing, 2011, p. 17). Esto tendría su máxima expresión en los mercados laborales generados tras la crisis de 2008 - especialmente los modelos de negocio asociados a tecnologías digitales (De Rivera et al., 2017 , p. 24) - donde se vería en buena medida resuelta de manera desfavorable para buena parte de los trabajadores y trabajadoras la contraposición entre seguridad y flexibilidad. Así, señala que la población sujeta a las lógicas neoliberales que llevarían a un escenario de precariedad habrían perdido las formas de seguridad relacionadas con el trabajo que habrían intentado implantarse tras la Segunda Guerra Mundial: Seguridad en el mercado laboral, seguridad en el empleo, seguridad en el puesto de trabajo, seguridad en el trabajo, seguridad en la reproducción de las habilidades, seguridad en los ingresos y seguridad en la representación (Standing, 2011).

Los discursos de la flexibilidad implican ciertas amenazas y desafíos para el trabajo. A pesar de los posibles beneficios de la economía de plataformas a la hora de instalarse en la lógica de la flexibilidad, se podría dar una sobre estimación de sus beneficios. Como señala De Stefano (2016, p. 5, nuestra traducción):

Si bien es cierto que la mayoría de los puestos de trabajo de la gig economy tienen un horario flexible, esto no dice mucho sobre la sostenibilidad general de estos acuerdos: la competencia entre los trabajadores, que en algunos casos se extiende a una dimensión global a través de Internet (Agrawal et al. 2013; Kingsley et al., 2014), empuja las compensaciones hacia abajo, de modo que las personas se vean obligadas a trabajar muchas horas y a renunciar a una buena cantidad de flexibilidad para obtener ingresos reales (Aloisi, 2015; Cherry, 2009, Eurofound, 2015, Felstiner, 2011). Además, los trabajos pueden ser publicados o necesitan ser ejecutados principalmente a ciertas horas del día: esto puede limitar significativamente la flexibilidad a la hora de fijar las horas de trabajo.

La aparición y proliferación de las plataformas digitales está íntimamente relacionada con las situaciones en el mercado de trabajo tradicional, ajeno a las plataformas. En este sentido Srnicek (2017, p. 74) afirma que "el mercado de trabajo tradicional que más se acerca al modelo de la plataforma austera es uno viejo y de baja tecnología: el mercado de los jornaleros [...], que se presentan en un lugar a la mañana con la esperanza de encontrar un trabajo por el día". A esta afirmación añade que:

Estas compañías son tristemente célebres por la subcontratación de sus trabajadores. En los Estados Unidos, estas plataformas entienden legalmente a sus trabajadores como "contratistas independientes" más que como "empleados". Esto les permite a las empresas ahorrar alrededor del $30 \%$ en costos laborales mediante un recorte de las prestaciones, las horas extras, los días por enfermedad y otros costos. Implica también deslocalizar los costos de training, dado que el training solo está permitido a los empleados; y este proceso llevó a formas alternativas de control, vía sistemas de reputación, que a menudo transmiten las tendencias racistas y de género de la sociedad (Srnicek, 2017, pp. 72-73).

Sin duda, algo que está caracterizando a las economías digitales, como ya ha sido señalado anteriormente, es la aparición y extensión de formas de empleo atípicas, diferentes a las formas tradicionales de empleo estable. Como apunta Miguélez (2018, pp. 151-152)

"frecuentemente la relación del trabajador con la empresa se sale de los cánones clásico, es decir, contrato, salario, algún tipo de estabilidad, para adquirir características propias de asociación o trabajo por cuenta propia”.

\subsection{El papel de la tecnología en las transformaciones de la economía y su rol en la situación de emergencia de las plataformas digitales}

Durante la crisis económica han hecho aparición y se han consolidado una serie de elementos tecnológicos que han afectado a la composición y han supuesto algunas transformaciones y cambios en el mercado de trabajo. Estas tecnologías han implicado transformaciones en múltiples dimensiones del empleo, como la organización del trabajo, la forma en la que se llevan a cabo las actividades laborales o cambios en las exigencias de los puestos de trabajo a la hora del manejo de ciertas tecnologías, pero también, la progresiva aparición de algunos puestos de trabajo y la sustitución o desaparición de otros. Sobre los trabajos que aparecen o desaparecen con las nuevas economías digitales, Miguélez (2018, p. 152) plantea que "[...] cabe preguntarse sobre el riesgo de destrucción de empleo que las nuevas tecnologías - robots, plataformas digitales, inteligencia artificial, etc. - pueden provocar en España, pero también sobre los nuevos empleos que pueden aparecer" ya que se daría una carencia de marcos regulatorios para este tipo de empleos.

Degryse (2016) señala que las mejoras técnicas y de las capacidades tecnológicas, como el aumento de la velocidad de las redes de internet, la extensión y sofisticación de las prácticas de recopilación, explotación y análisis masivos de datos y el contundentemente extendido acceso a los terminales móviles han sido fundamentales en la generación de nuevas perspectivas para el mercado laboral. Sin embargo, como señala Srnicek (2017, p. 74), "una importante razón por la cual los teléfonos móviles se han vuelto esenciales en el desarrollo de los países es que ahora son indispensables en el proceso de encontrar trabajo en los mercados de trabajo informal". De la misma manera, Degryse (2016, pp. 7-36) afirma que, ante esta extensión de las capacidades tecnológicas, se habría venido dando un progresivo cambio en las relaciones laborales en las que habría una tendencia a la aparición de formas de empleo atípi- 
cas. Asimismo, estas incorporaciones tecnológicas habrían supuesto también la introducción de mecanismos de control, individualización del trabajo y desprotección a través de los mecanismos de rating y rankings en las plataformas digitales (De Rivera, Gordo y Cassidy, 2017, p. 26; Gandini, 2018, p. 11). Así, en investigaciones como la que aquí se presenta se ha señalado que los mecanismos de rating funcionan sobre mecanismos de presión y control sobre los trabajadores (Goods, Veen y Barratt, 2019), más allá de ser meros mecanismos para la mejora de los servicios ofrecidos por las plataformas.

Por último, es conveniente apuntar a que, ante esta situación, son múltiples las investigaciones (Berins, Dubal y Carter, 2017; De Stefano, 2016; Garben, 2017; Miguélez, 2018; Vandal, 2018; Zamponi, 2019) que señalan que las apariciones de estas formas de trabajo sujetas a la incorporación de tecnologías supondrían también una amenaza para las relaciones laborales y la organización y representación de trabajadores y trabajadoras, suponiendo un reto para el sindicalismo, al menos en sus formas más tradicionales (Fita y Goerlich, 2017, p. 44).

\section{Objetivo y planteamiento de la investigación}

El objetivo de este trabajo es el de analizar cómo se reproducen algunos de estos elementos previamente señalados en la realidad del trabajo de plataformas, en concreto, aquellas dedicadas al reparto y la mensajería. El punto de partida es que las realidades generadas por estos elementos en la economía y el mercado de trabajo tendrían consecuencias más allá de algunos lugares comunes como son lo innovador en estas formas de trabajo, la proliferación de puestos de trabajo gracias a las plataformas digitales o la imparcialidad de este tipo de plataformas en términos de generación de empleo y modelos económicos, planteando que es necesario cuestionarse estas realidades. Así, se plantea que el momento de crisis en el que se da el desarrollo de estas plataformas y la manera en la que lo hacen implican diferentes formas de precariedad, inseguridad en el ámbito del trabajo y carencia de estabilidad contrapuestas al discurso de innovación y una mirada desproblematizada de la flexibilidad que proponen las plataformas.

Para ello, esta investigación se basa en una serie de doce entrevistas realizadas durante enero y junio de 2019 a repartidoras y repartidores, de una duración que oscilaba entre los 45 minutos y 1 hora y 15 minutos, todas ellas en Madrid. Estas entrevistas fueron realizadas de manera semiestructurada con el objetivo de encontrar en sus discursos las claves del trabajo en las plataformas digitales de reparto. La comunicación con los repartidores y repartidoras entrevistados se ha realizado a través de un primer contacto con dos organizaciones de repartidores y repartidoras. A través de este contacto, diferentes repartidores y repartidoras manifestaron su interés en llevar a cabo las entrevistas e hicieron difusión entre compañeros y compañeras para la puesta en contacto y realización de entrevistas.

Los perfiles de las repartidoras y repartidores comprenden una variabilidad basada en características sociales como la procedencia, el género o la edad; las trayectorias laborales atendiendo a quienes hubieran trabajado en el reparto y mensajería de manera previa; y a quienes trabajasen o hubiesen trabajado en otro tipo de empresas diferentes a las grandes plataformas digitales.

Los perfiles seleccionados responden a encontrar cierta variabilidad en los discursos y las percepciones que estarían presentes entre quienes trabajan en las plataformas digitales. Sin embargo, es necesario tener en cuenta de antemano que estos perfiles no se corresponderían con una muestra precisa de la realidad de quienes trabajan en las plataformas de reparto de comida, ya que según los discursos de los mismos entrevistados y entrevistadas y algunos de los estudios realizados en otros ámbitos llevaría a que se diera una infrarrepresentación de la población migrante de origen latinoamericano entre los entrevistados y entrevistadas y, en contraste, estar sobredimensionada la población de origen español.

Solo han sido tenidas en cuenta para esta investigación quienes hubieran trabajado o se encontraran trabajando con alguna o varias de las grandes plataformas que funcionan o han funcionado en España (Deliveroo, Glovo, Stuart, UberEats o Take Eat Easy). De éstas, nueve de los entrevistados eran hombres y tres mujeres, cuatro habían nacido en diferentes países de América Latina, dos en Italia y el resto (seis) en España. De entre las personas entrevistadas tan solo cuatro seguían trabajando en el momento de realización de la entrevista en alguna o varias de las grandes plataformas de reparto, dos habían tomado otras alternativas laborales fuera del sector y seis seguían trabajando en el ámbito del reparto y la mensajería en diferentes tipos de empresas, como cooperativistas o contratados en otras compañías diferentes a las grandes plataformas. De entre las personas entrevistadas una gran proporción tenía formación en campos muy diferentes al de la logística y no habría tenido experiencia previa en el sector.

La selección de la muestra ha estado basada en la búsqueda de variabilidad y abarcar los distintos discursos que existirían entre quienes trabajan en estas plataformas, y no tanto a un criterio de proporcionalidad de los perfiles más habituales en este caso de estudio.

Preeminentemente el perfil de quienes reparten en estas grandes plataformas muestran una tendencia a estar masculinizado y estar asociado a población migrante, especialmente de origen latinoamericano, como han señalado los entrevistados y entrevistadas en diferentes ocasiones, el reciente informe publicado por la UGT $(2019$, p. 56) y como han señalado investigaciones a nivel europeo (Dufresne, 2019, p. 15).

La utilización de la entrevista como metodología de investigación está basada en una decisión pragmática dada la utilidad que ofrece para esta investigación la recopilación de unos discursos de los que 
no se suponían premisas previas, especialmente en un ámbito complejo como el del trabajo en un ámbito relativamente reciente y complejo que es entendido como un elemento de cambio en las sociedades contemporáneas. Dados los objetivos de esta investigación, centrados en las percepciones, delimitar y medir la relevancia de los principales problemas que atraviesan el conflicto desde las perspectivas de quienes trabajan o han trabajado en este tipo de plataformas, la entrevista como metodología para el acercamiento a estas cuestiones ha sido considerada la más útil. Siendo el objetivo de esta investigación analizar en qué medida son reales en el desarrollo del trabajo en estas plataformas por quienes lo llevan a cabo, las entrevistas han permitido acceder a los discursos que desgranan realidades concretas en este sentido, como se desarrollará posteriormente. Asimismo, y al margen de la discusión sobre la pertinencia de unas u otras técnicas, es necesario señalar que otras vías de abordaje de esta cuestión a través del análisis de estudios como encuestas o análisis estadísticos no resultan posibles en el caso de España en la actualidad. Debido al carácter difuso y novedoso, así como el todavía relativamente bajo número de personas que trabajan en este ámbito, de estas formas de trabajo no es posible debido a la ausencia de datos disponibles. Buena parte del fundamento de esta investigación estaría basado en la referencia a otras investigaciones como la realizada por Goods, Veen y Barratt sobre la calidad del trabajo en las plataformas de reparto de comida en Australia (2019).

\section{Resultados. Los discursos en el trabajo en las plataformas de reparto}

\subsection{Precariedad}

Como se señalaba anteriormente las plataformas digitales nacen en un momento de vulnerabilidad social y desprotección. La inseguridad generada en los mercados de trabajo habría empujado a los sectores más precarizados de la sociedad hacia una situación de indefensión y de búsqueda de realidades laborales al margen de las formas de trabajo más estable tras la crisis. Entre los discursos recogidos de quienes hubieran trabajado o estuvieran trabajando para plataformas de reparto encontramos diferentes dimensiones de la precariedad en el trabajo.

Así lo vemos reflejado en los discursos planteados por los repartidores y repartidoras de las plataformas digitales, quienes señalaban, por un parte, la llegada a las plataformas de reparto tras una experiencia laboral normalmente precarizada y habitualmente breve, tanto en el sector de reparto como fuera de éste: "Llegué ahí por lo que te vendían, lo que te vendían de autonomía, lo que te vendían de pasta también. No sé muy bien por qué, yo estaba buscando trabajo y pues no sé llegue a Deliveroo de rebote" (INT11).

Esto también se ve reflejado en el hecho de que una gran proporción de las personas entrevistadas vendría de trayectorias laborales y formativas diferentes a la actividad que venían desarrollando en las plataformas de reparto, si bien algunos y algunas de las que fueron entrevistados y entrevistadas habían trabajado con anterioridad en el reparto de comida o la mensajería. Y, por otro lado, la llegada al trabajo en plataforma como única salida laboral dadas las características curriculares y trayectorias vitales, por ejemplo, en el caso de la población migrante.

En algunos de los discursos analizados puede percibirse también cómo se ha dado cierta frustración de las expectativas en el trabajo de las plataformas de reparto de comida. Así, entre los discursos de las personas migrantes entrevistadas encontramos algunos elementos comunes y repetidos. Entre aquellas que provienen de países con idiomas diferentes al castellano (entre las personas entrevistadas fundamentalmente de países europeos) señalan la adecuación y facilidad de acceso a este tipo de trabajos. Mientras que por otro lado, quienes vienen de países de Latinoamérica, que comparten el idioma, la predisposición al trabajo en las plataformas de reparto se debe a motivos como la dificultad para la homologación de títulos de formación, lo que dificulta la incorporación al mercado laboral en ámbitos no cualificados en los que no tienen experiencia previa y la existencia de redes familiares y de amistades que informan sobre esta alternativa laboral y facilitan la incorporación al conocer el proceso por estar o haber estado trabajando con anterioridad.

Asimismo, otro de los elementos que podemos encontrar en los discursos de quienes han trabajado o trabajan en las grandes plataformas digitales es la tendencia a aumentar el número de horas trabajadas para aumentar las ganancias. Como señala otro repartidor:

[...] como no soy un muchachito no puedo trabajar doce horas al día todos los días, además me parece una locura porque hay gente que lo hace, pero me parece una locura trabajar en esto en la calle manejando doce horas al día porque en algún momento te va pasar algo. Conozco a un chico venezolano que ya ha tenido dos accidentes porque estaba cansado [...]. Se partió la clavícula la última vez porque estaba cansado. Yo le dije '¿te dormiste?', 'no', pero estaba en blanco. Se metió contra la bifurcación en la M-30 se metió contra la defensa y terminó allá y la moto terminó allá picada en dos. A él no le pasó nada: milagro. Pero es porque estaba cansado (INT12).

Esto supondría también la ruptura con el concepto de seguridad en el trabajo, dado que debido a la carencia de una vinculación estable y de carácter contractual con las diferentes empresas llevaría a que recayera la responsabilidad sobre el repartidor o la repartidora de una parte importante de la seguridad física.

Asimismo, encontraríamos en estos discursos otras muestras de disconformidad con las características de trabajar para las grandes plataformas de reparto en España, siendo una de las más extendidas el pago de los servicios. Si bien podría percibirse un descontento general con la cantidad establecida por los servicios prestados o del precio del trabajo, ha- 
brían sido frecuentes las críticas a la complejidad del establecimiento de estos precios y, sobre todo, la imposición de estos precios marcados por las empresas. Esta manifestación de disconformidad estaba frecuentemente asociada a la situación de la relación con la empresa, planteando que siendo trabajadores y trabajadoras autónomos tenían una incapacidad total a la hora de marcar los precios por servicio o tarea realizados. En otras palabras, los entrevistados y entrevistadas encontrarían contradicción entre el hecho de ser autónomos y las obligaciones derivadas de ello y la falta de autonomía a la hora de establecer cuáles serán sus ingresos o los horarios en los que trabajaran, ya que estos son marcados por la plataforma. Esto es algo que se ve mayoritariamente reflejado en los discursos de quienes han trabajado o trabajan en estas grandes plataformas de reparto. Como ejemplo se puede observar cuál es una de las principales problemáticas para un repartidor:

Para mí ha sido el mejor trabajo que he tenido a nivel trabajo, pero a nivel de condiciones era una putada, porque claro, el seguro te lo tenías que pagar tú. $\mathrm{O}$ sea, es como lo de ser autónomo, ¿no? Los medios para ganar el dinero lo tenías que poner tú, pero luego tú ese dinero no lo podías rentabilizar. O sea, yo entiendo que si soy autónomo con una hora de mi tiempo gano 10 euros, pero a lo mejor en 10 meses currándomelo con una hora mi tiempo gano mucho más dinero. Pero ahí no (INT7).

\subsection{Flexibilidad}

Llegados a este punto, lo que relacionaría los dos elementos de análisis de este estudio sería el concepto de flexibilidad. Además de los puestos que se generan en el mercado de trabajo gracias a las plataformas digitales de reparto, la flexibilidad se ha convertido en uno de los grandes argumentos a favor de la economía de plataforma y reclamo de las compañías para el reclutamiento de repartidores y repartidoras.

Como se planteaba con anterioridad, en el marco de generación de formas laborales de precariedad desde una visión neoliberal del trabajo, los que ponen en marcha estas prácticas desarrollan un discurso sobre la flexibilidad asociados a conceptos como libertad o autonomía que chocarían con las percepciones de quienes trabajan en las plataformas. Así, en la web de Deliveroo presentan el trabajo con esta plataforma de la siguiente manera:

Deliveroo está orgulloso de ofrecer trabajo flexible y bien remunerado, y los riders son el corazón de Deliveroo. [...] Deliveroo se preocupa por los riders y por ello, es la primera empresa de la economía bajo demanda que ha decidido poner fin al límite entre flexibilidad y seguridad que impera en el derecho laboral ${ }^{2}$.

En el mismo sentido, en una entrevista en la sección Retina de El País a Òscar Pierre, fundador de Glovo, ante la pregunta, "¿Crees que tendría que me-

https://deliveroo.es/es/about-us jorar la legislación de los autónomos?" contesta lo siguiente:

Sí, la sociedad está cambiando. El tema de la flexibilidad se infravalora pero es muy importante. Poder decidir cuándo, cómo y dónde trabajas es fundamental. Estoy seguro de que cada vez habrá más gente que dirá que no quiere tener jefes, no querrá tener horarios, y tendrá diferentes vías de ingresos: hará clases, trabajará como glover, pondrá su piso en Airbnb... Esta es la tendencia del futuro. Además, es muy positiva la cantidad de talento y riqueza que generan estas plataformas en el plano tecnológico (En Catà Figuls, 2018).

De estas pequeñas muestras del discurso de dos de las más importantes plataformas de reparto en España se pueden extraer dos elementos claves. Por una parte, la flexibilidad como uno de los principales atractivos a la hora de trabajar en estas plataformas, enmarcado en un discurso de adaptación a las nuevas realidades de las necesidades de quienes buscan empleo. Por otro lado, la pretensión de transformar el mercado laboral y las economías hacia un modelo concreto menos regularizado.

Esto lo vemos reflejado en los discursos de quienes han trabajado para estas plataformas: "[...] la parte negativa es eso. Que toda esa supuesta autonomía que luego no tenías, pero a mí el tipo de trabajo me gustaba mucho." (INT11). Como planteaba una repartidora de manera más extensa:

Y sobre todo y más importante, cuando estas empresas te venden todo el rollo de la flexibilidad, ¿no?, te venden como la idea de una persona joven que a lo mejor está estudiando, pero en realidad si lo piensas la mayoría de la gente que trabaja en estas aplicaciones es gente que no tiene otra alternativa laboral no es flexibilidad, o sea, la flexibilidad no es su parte positiva sino que es su parte negativa. Es la parte de: tengo que estar disponible en la mayor cantidad de turnos posibles, hacer la mayor cantidad de pedidos posibles (INT8).

Vemos aquí reflejado, algo muy presente en los discursos de repartidores y repartidoras, la relación de dependencia de estas grandes plataformas contrapuesta a una falta de seguridad en su forma principal de su fuente de ingresos.

\subsection{Innovación y transformación}

Entre quienes trabajan en estas plataformas encontramos discursos de normalización de su realidad cuando ésta es atendida desde una óptica altamente problematizada y complejizada desde el ámbito del estudio académico y la visión periodística. En lo referido al campo del desarrollo del trabajo en un sentido amplio, se percibe una normalización y equiparación con otras realidades laborales no sujetas a la atención recibida por el carácter innovador o transformador de las plataformas digitales. Habría una traslación en los discursos desde unas realidades laborales complejas intervenidas por la tecnología a una normalidad en el desarrollo del trabajo en el que está mediado por ciertas peculiaridades como las aplicaciones o lo que 
está siendo planteado como una transformación integral en el ámbito del trabajo, es simplemente visto como realidades laborales con malas condiciones y precariedad.

Adigital, la Asociación Española de la Economía Digital a la que pertenecen Deliveroo, Glovo o Uber, se presenta como "una asociación de empresas innovadoras y transformadoras que creen y trabajan por el desarrollo de la economía basada en Internet, el Medio Digital y las Nuevas Tecnologías"’. Así, es habitual encontrar algunos discursos en el ámbito periodístico y empresarial en el que se plantean que la proliferación de nuevas realidades empresariales mediadas por tecnología como son las plataformas digitales, traen consigo un carácter innovador y colaborativo. Sin embargo, es necesario cuestionarse a propósito de estos nuevos modelos y sobre sus implicaciones.

Otra cuestión controvertida y puesta en discusión es el carácter colaborativo de las plataformas digitales y la presunción de éstas como entidades dentro de la llamada economía colaborativa. Así, Sharing España, se presenta como:

Un colectivo que nace dentro de la Asociación Española de la Economía Digital (Adigital) y que agrupa diferentes empresas de la economía colaborativa, bajo demanda y de acceso, con el objetivo de analizar y divulgar el impacto que estas nuevas economías y los modelos basados en plataformas tienen en el desarrollo socioeconómico y en la sostenibilidad. Para Sharing España (SHES) la economía colaborativa representa la aparición de nuevos modelos empresariales y de consumo en los que gracias a las nuevas tecnologías se accede a bienes y servicios más eficientes y participativos ${ }^{4}$.

De entre los discursos recopilados, se puede encontrar con frecuencia una visión contrapuesta a esta idea de innovación, en la que el trabajo de reparto en las plataformas digitales ha sido la continuación de trayectorias laborales de precariedad. Así, reconocen el uso de los terminales móviles y funcionamiento a través de las plataformas, más como una forma de organización y control sobre su trabajo que como un elemento realmente innovador en el trabajo. En este sentido un exrepartidor de una de las grandes plataformas de trabajo, quien constituyó junto a otros compañeros y compañeras una cooperativa de reparto en Madrid, plantea que:

Después de la huelga, de las protestas de 2017, se creó un grupo con muchas ideas de crear algo independiente, más ético, más comprometido y económicamente más digno que trabajar para las grandes plataformas. Nuestro objetivo al montar una cooperativa era en principio el de emanciparnos de una condición de precariedad [...] pero también el de cooperar. Cooperar con otras cooperativas y cooperar con los restaurantes (INT3).

La intencionalidad y las lógicas de las plataformas a la hora de constituir una iniciativa de negocio para el reparto y la mensajería generan conflictos. Como

\footnotetext{
https://www.adigital.org/quienes-somos/

http://www.sharingespana.es/quienes-somos/
}

posible respuesta a esta problemática se han planteado fórmulas que se contrapongan a la realidad de precariedad que está asociada a las grandes plataformas, poniendo en valor elementos como la cooperación, colaboración y mejora de las condiciones laborales.

\section{Conclusiones}

Una de las cuestiones más importantes para comprender el desarrollo y expansión del capitalismo de plataforma, así como las formas y maneras concretas en las que estas tienen lugar, es que su evolución se da en un momento dado y preciso. Pueden ser considerados como fundamentales e íntimamente relacionados con este momento los tres elementos contextuales señalados: la crisis como momento de emergencia de las plataformas de reparto, las condiciones del mercado de trabajo en el que funcionan y la incorporación tecnológica como determinante en las condiciones laborales.

A través de las entrevistas realizadas se puede observar cómo es necesario analizar en profundidad las nuevas realidades que se generan con el trabajo de plataforma. La proliferación de formas de empleo atípico (De Stefano, 2016; Garben, 2017; Miguélez, 2018) es una cuestión crucial a la hora de analizar qué ha significado la incorporación del trabajo en plataformas laborales al mercado laboral. Y es que la crisis ha sido un momento propicio para la proliferación de las plataformas digitales como modelo de transformación de las formas de empleo (Laval y Dardot, 2013; Srnicek, 2017).

Distintos autores (Laval y Dardot, 2013; Sadin, 2018; Srnicek, 2017) tratan qué han supuesto, en términos de erosión de los sistemas de bienestar y seguridad en términos globales y generales, las dinámicas asociadas a la introducción de las economías de plataforma. En los resultados encontramos que esto tiene consecuencias en lo concreto para quienes trabajan en este tipo de plataformas, quienes ven en la pérdida de estabilidad laboral y vital, añadida una erosión de sus garantías sociales asociadas al trabajo en relación con las formas de contratación y relación con las grandes plataformas de carácter atípico y en las que se esquivan los sistemas de protección de trabajadores y trabajadoras.

Asimismo, como ha sido mostrado en los discursos encontrados en esta investigación, en las dinámicas en el desarrollo del trabajo cotidiano en las plataformas de reparto de comida encontramos contradicciones con las premisas de flexibilidad que plantean los discursos de las plataformas digitales (De Stefano, 2016).

Con respecto al carácter innovador en el ámbito del trabajo, no se habrían encontrado discursos entre repartidores y repartidores que indiquen que las herramientas tecnológicas implicadas en el desarrollo de su trabajo impliquen una mejora sino un elemento de presión bajo el desarrollo de su trabajo en los aspectos concretos de la actividad laboral, con distintas reticencias con respecto a las implicaciones de su trabajo (De De Rivera et al., 2017; Degryse, 2016; Gandini, 2018; Goods, Veen y Barratt, 2019; Srnicek, 2017). 
Por último, como se ha visto reflejado en los discursos de los repartidores y las repartidoras la realidad del trabajo de plataformas está ligado en la actualidad con ciertas formas de precariedad que muestran incoherencias con los discursos de flexibilidad y adaptación a los mercados de trabajo de las plataformas de reparto.

El momento actual de expansión de las plataformas digitales requiere de estudios pormenorizados de las distintas problemáticas que se le asocian, especial- mente si tenemos en cuenta las complejidades que implican a nivel jurídico, técnico y de organización del trabajo. Asimismo, las diferentes realidades en las que se desarrollan implican la necesidad de tener muy en cuenta los contextos en los que se desarrollan para medir las consecuencias sobre la realidad del trabajo en las próximas décadas, como son los mercados laborales, las culturas sindicales o de movilización en los diferentes escenarios y las tendencias del consumo en un marco tendente hacia lógicas neoliberales.

\section{Referencias}

Alonso, L. E., Fernández, C. J., y Ibáñez, R. (2016). De la moral del sacrificio a la conciencia de la precariedad: un análisis cualitativo de los discursos sobre la evolución de la crisis en España. Política y sociedad, 53(2), 353-379.

Bouffartigue, P. (2015). «Précarité»: de quoi parle-t-on? Second séminaire Tramed «Les problématiques du travail dans l'espace euro-méditerranéen en crise: précarité et jeunes générations», octubre, Nápoles.

Busch, C., Schulte-Nölke, H., Wiewiórowska-Domagalska, A. y Zoll, F. (2016). The Rise of the Platform Economy: A New Challenge for EU Consumer Law? Journal of European Consumer and Market Law. 1, 3-10.

Calderón, J. Á. y López Calle, P. (2010). Transformaciones del trabajo e individualización de las relaciones laborales. La emergencia de nuevas formas de resistencia al trabajo. Estudios de la Fundación, 24, 3-16.

Catà Figuls, J. (2018). Economía digital. El fundador de Glovo: "En el futuro la gente tendrá múltiples vías de ingresos", El País Economía, Retina. 1 de mayo, https://retina.elpais.com/retina/2018/04/30/tendencias/1525088881_394696. html

Conaty, P., Bird, A. y Ross, C. (2018). Working Together. Trade union and co-operative innovations for precarious workers. Manchester: Co-operatives UK.

De Rivera, J.; Gordo, A.J. y Cassidy, P. (2017). La economía colaborativa en la era del capitalismo digital. Redes.com: revista de estudios para el desarrollo social de la Comunicación, 15, 20-31.

De Stefano, V. (2016). The Rise of the 'Just-in-Time Workforce': On-Demand Work, Crowd Work and Labour Protection in the 'Gig-Economy', Bocconi Legal Studies Research Paper (2682602). http://dx.doi.org/10.2139/ssrn.2682602

Degryse, C. (2016). Digitalisation of the economy and its impact on labour markets. Bruselas: ETUI.

Dufresne, A. (2019). Les acteurs de la lutte: collectifs et syndicats Vers de nouvelles identités collectives? Gresea Échos, 98, 14-21.

Fita, F. y Goerlich, J. M. (2017). Sindicalismo y acción sindical en el siglo XXI: crisis económica y transformación del modelo productivo. Arxius de ciènces socials, 36-37, 37-48.

Florisson, R. y Mandl, I. (2018). Platform work: Types and implications for work and employment - Literature review, Working paper WPEF18004. Dublin: Eurofound.

Gandini, A. (2019). Labour process theory and the gig economy. Human Relations, 76(6), 1039-1056.

Garben, S. (2017). Protecting Workers in the Online Platform Economy: An overview of regulatory and policy developments in the UE. Luxembourg: Publications Office of the European Union.

Gil, J. (2018). ¿Qué son las economías colaborativas?. Papeles de relaciones ecosociales y cambio global, 141, 47-60.

Goods, C.; Veen, A. y Barratt, T. (2019). "Is your gig any good?" Analysing job quality in the Australian platform-based food-delivery sector. Journal of Industrial relations, 61(4), 502-527.

Howcroft, D. y Bergvall-Kåreborn, B. (2019). A Typology of Crowdwork Platforms. Work, Employment and Society, $33(1), 21-38$.

Laval, C. y Dardot, P. (2013). La nueva razón del mundo. Ensayo sobre la sociedad neoliberal. Barcelona: Gedisa Editorial.

Lietaert, M. (2017). Homo Cooperans 2.0. Por una economía colaborativa desde el cooperativismo. Barcelona: Icaria.

Miguélez, F. (2018). Revolución digital y futuro del empleo. Anuario IET de Trabajo y Relaciones Laborales, 5, 147-161. https://doi.org/10.5565/rev/aiet

Moral Martín, D. y Brunet, I. (2018). La imagen del sindicato en el siglo XXI a la luz de su contestación por los Nuevos Movimientos Sindicales. Sociología del Trabajo, 93, 307-326.

Rogers, B. (2016). Employment Rights in the Platform Economy: Getting Back to Basics. Harvard Law y Policy Review, Forthcoming, Temple University Legal Studies Research Paper No. 2015-33. http://dx.doi.org/10.2139/ssrn.2641305

Sadin, É. (2018). La silicolonización del mundo. La irresistible expansión del liberalismo digital. Buenos Aires: Caja Negra.

Santos, A. y Martín, P. (2012). La juventud española en tiempos de crisis. Paro, vidas precarias y acción colectiva. Sociología del trabajo, 75, 93-110. 
Scholz, T. (2016). Platform Cooperativism. Challenging the Corporate Sharing Economy. New York: Rosa Luxemburg Stiftung.

Srnicek, N. (2018). Capitalismo de plataformas. Buenos Aires: Caja Negra.

Standing, G. (2011). El precariado. Una nueva clase social. Barcelona: Pasado y Presente.

UGT (2019). El trabajo en las plataformas digitales de reparto. Estudios, 1.

Vallas, S. P. (2019). Platform Capitalism: What is at Stake for Workers? New Labor Forum, 28(1), 48-59

Vandaele, K. (2018). Will trade unions survive in the platform economy? Emerging patterns of platforms workers' collective voice and representation in Europe. Working Paper 2018.5 Bruselas: european trade union institute (ETUI). 